\title{
Modelo TPACK y metodología activa, aplicaciones en el área de matemática. Un enfoque teórico
}

\author{
Fecha de recepción : 23-02-2021 • Fecha de aceptación: 13-04-2021 • Fecha de publicación: 10-05-2021
}

\author{
María Fernanda Rodríguez Solís ${ }^{1}$ \\ Pontificia Universidad Católica del Ecuador - Sede Ambato \\ maria.f.rodriguez.s@pucesa.edu.ec \\ https://orcid.org/0000-0003-0094-9235 \\ Santiago Alejandro Acurio Maldonado² \\ Pontificia Universidad Católica del Ecuador - Sede Ambato \\ sacurio@pucesa.edu.ec \\ https://orcid.org/0000-0001-7069-9206
}

\section{Resumen}

El artículo investigativo de revisión sistemática plantea identificar las estrategias innovadoras tecnológicas mediante el modelo TPACK, más la metodología activa en los docentes de los terceros años de educación general básica para la catedra de restas con reagrupación. Con la base teórica del trabajo de Contreras, Tristancho, \& Fuentes (2017); quienes consiguieron evolucionar el tipo de enseñanza, con una mayor intervención de los estudiantes, fortaleciendo un aprendizaje distinto y puntual, acrecentando el análisis y desenvolvimiento en matemática, con el objetivo de trabajar con el modelo pedagógico y tecnológico de contenidos (TPACK). El sustento metodológico elegido fue la investigación cualitativa en donde se aplica el modelo de investigación documental de Hoyos (2000), el cual resalta el proceso de las fases preparatoria, descriptiva, interpretativa por núcleo temático, de construcción teórico global y de extensión. Como conclusión se establece que las herramientas didácticas digitales facilitan nuevos mecanismos de conocimiento, la mejora de los tiempos de aprendizaje y los niveles de efectividad en la resolución de problemas. Se expone varios tipos de recursos digitales los mismos que pueden ser implementados dentro y fuera de las aulas de clase según la necesidad. 
Palabras clave: Fases, modelo TPACK, metodología activa, matemática, restas con reagrupación, revisión sistemática de literatura.

\section{Abstract}

The investigative article of systematic review proposes to identify the innovative technological strategies through the TPACK model, plus the active methodology in the teachers of the third years of basic general education for the chair of subtraction with regrouping. With the theoretical basis of the work of (Contreras, Tristancho, \& Fuentes, 2017); who managed to evolve the type of teaching, with a greater intervention of the students, strengthening a different and punctual learning, increasing the analysis and development in mathematics, with the aim of working with the technological and content pedagogical model (TPACK). The methodological support chosen was qualitative research where the documentary research model of Hoyos (2000) is applied, which highlights the process of the preparatory, descriptive, interpretative phases by thematic nucleus, of global theoretical construction and extension. As a conclusion, it is established that digital teaching tools facilitate new knowledge mechanisms, the improvement of learning times and levels of effectiveness in solving problems. Various types of digital resources are exposed, which can be implemented inside and outside the classrooms as needed.

Keywords: Phases, TPACK model, active methodology, mathematics, subtraction with regrouping, systematic literature review. 


\section{Introducción}

El modelo TPACK (Tehnological Pedagógica Content Knowledge), es un modelo de enseñanza aprendizaje, que domina tres tipos de Conocimientos, Pedagógico, Contenidos y Tecnológico (Gómez, 2015). Así, el Conocimiento Pedagógico (PK): concerniente con los modelos educativos, los objetivos de aprendizaje y particularidades adecuadas de los procesos educativos que se solicita para iniciar una enseñanza de calidad. El Conocimiento de Contenidos (CK): Racionado con las temáticas adecuadas de cada área de estudio, sus objetivos de aprendizaje y contenidos curriculares que se deben compartir dentro del aula. El Conocimiento Tecnológico (TK):

Concerniente con las destrezas en la aplicación de herramientas de tecnología útiles dentro de los métodos educativos (Arévalo, García, \& Hernández, 2019). Este modelo es la sociedad de diversas características que ayudan al aprendizaje de los estudiantes para una mejor comprensión del área de matemática, como al aprendizaje autónomo.

Como se observa, el modelo TPACK tiene tres grupos de competencias.

- Las competencias Tecnológicas Pedagógicas (TPK) que contienen las estrategias para trabajar dentro del aula con la tecnología proporcionándole dinámica al aprendizaje.

- Las Competencias Tecnológicas del Contenidos (TCK), donde se relaciona todos los contenidos de cada tema con la tecnología.

- Las Competencias Pedagógicas del Contenido (PCK), donde la Pedagogía se despliega con cada uno de los contenidos.

Las interacciones entre los tres grupos de competencias indicadas, van a crear a su vez nuevas competencias para la enseñanza aprendizaje del área de matemática como lo muestra (González, Montes, \& Guerrero, 2018). Las nuevas representaciones de educación hacen que los docentes investiguen nuevas estrategias de enseñanza, por eso es trascendental el conocimiento del modelo TPACK (Figura 1) que vincula todos los conocimientos para que el estudiante pueda deducir en el aprendizaje (Muñoz, Briceño, \& Hernández, 2016). 


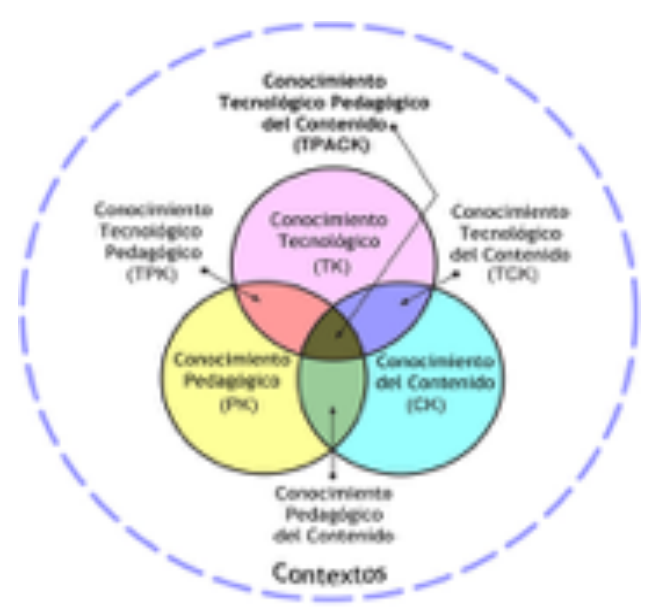

Figura 1. Modelo TPACK

Fuente: (González, Montes, \& Guerrero, 2018).

\subsection{Metodología activa}

La metodología activa es un conjunto de métodos planificados que ayudan al estudiante a obtener nuevos y diversos conocimientos como destrezas, motivándolo a tomar desafíos, decisiones y soluciones por sí mismos con su reflexión crítica y su capacidad mental; es decir, ayuda a solucionar inconvenientes. Para esto el estudiante difiere entre dos conocimientos, el conceptual y el procedimental, los conceptos básicos concernientes a los temas matemáticas, y los métodos expuestos para la práctica de los ejercicios a solucionar (Miro, 2006).

Esta desempeña un papel significativo en la educación creando nuevos escenarios para la enseñanza aprendizaje establecida dentro o fuera del aula de clase, donde se orienta una formación autónoma del estudiante, proporcionándole nuevos rumbos de protagonista y motivándolo para una nueva fase dentro de su vida personal. La metodología activa está cimentada en todas las áreas integrales con ámbito social; se basa, asimismo, en varias técnicas concernientes con el área, con un cronograma de trabajo organizado para que sea práctico en el aprendizaje de los estudiantes (Cueto, Morales, Burgueño, \& Medina, 2017).

En la Figura 2 se muestra una representación de esta metodología. 


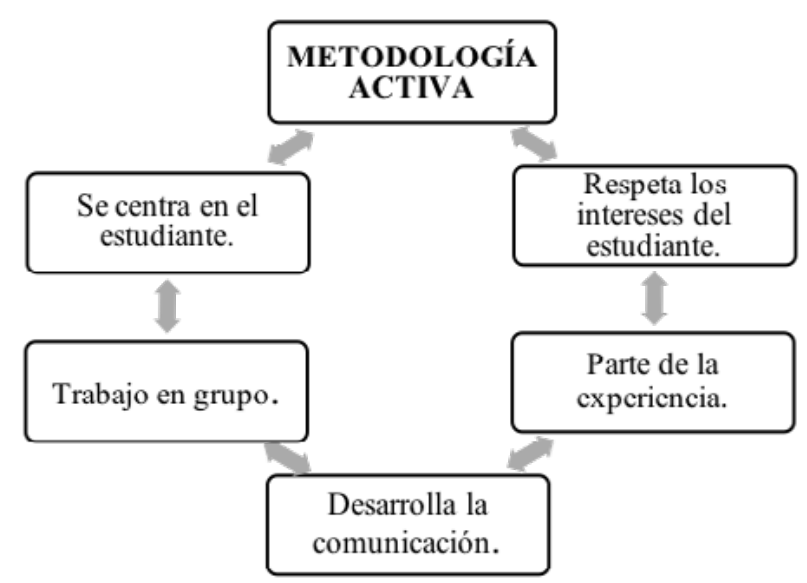

Figura 2. Metodología activa

Fuente: elaboración propia

Este proceso se caracteriza por mantener un respeto que se debe nutrir del interés de los estudiantes dando importancia al conocimiento del alumnado, esta metodología nace de la experiencia de los estudiantes para poder entender de mejor manera un nuevo tema a tratar, desarrolla la comunicación permitiendo el intercambio de ideas en la resolución de problemas, es importante en esta metodología el trabajo en equipo con los participantes, finalmente anotar que se centra en el estudiante como el ente más importante en el aprendizaje buscando implantar características prioritarias para ellos.

\subsection{TPACK en matemática}

Los dispositivos tecnológicos desde hace varios años han venido siendo un cambio fundamental en la sociedad, Deroche y otros (2015) manifiestan que los dispositivos tecnológicos brindan la posibilidad de optimizar la enseñanza en los estudiantes, de este modo la era digital que se vive en la actualidad es una representación estructurada y sistemática de plasmar la enseñanza y el aprendizaje de los estudiantes, ya que tecnológicamente están acoplados, rodeados de dispositivos tecnológicos y vinculados en una conectividad digital, brindando al estudiante un proceso significativo de aprendizaje autónomo y al mismo tiempo participativo.

Dentro de las aplicaciones usadas para un adelanto tecnológico se identifica el uso de aplicaciones móviles como lo expone Ferreira y Madrigal (2014), en la parte de ciencias de igual manera los estudiantes manejan laptops, tabletas, celulares en la actualidad, es más factible trabajar con esos medio tecnológicos para perfeccionar el aprendizaje significativo en el área de matemática como lo manifiesta Cenich, Araujo y Santos (2020) de la misma forma se utiliza móviles, tabletas. Mientras que también, en la experiencia de Salas (2017), se muestra la importancia de emplear la aplicación RAPTOR ${ }^{1}$, que ayuda al conocimiento de la matemática, igualmente la aplicación Facebook.

1 RAPTOR es un entorno de programación basado en diagramas de flujo, diseñado específicamente para ayudar a los estudiantes a visualizar sus algoritmos y evitar el equipaje sintáctico. Los programas RAPTOR se crean visualmente y se ejecutan visualmente al rastrear la ejecución a través del diagrama de flujo (Salas, 2017). 


\subsection{Aplicaciones didácticas digitales}

Dentro de las técnicas de la ejecución digital en las instituciones educativas para perfeccionar el aprendizaje, se ve la red Red Edusar que es una red de televisión que se incorpora al proceso educativo. Otros estudios manifiestan que la inclusión del computador a la educación origina una mejor enseñanza como recurso digital.

Las aplicaciones móviles, en la actualidad son una forma innovadora de llegar al aprendizaje de los estudiantes, donde una aplicación para un dispositivo puede ser una herramienta aceptada; y más precisa si se lo puede abrir en el computador, como lo indica Thomas, Dapoto \& Pesado (2017). De la misma forma el mobile learning o el aprendizaje móvil para el área de Matemática es ventajoso también para el adelanto social, constructivista y conductual ya que los estudiantes con el uso de una aplicación móvil estarán trabajando en estos ambientes.

Según Jiménez, Mora y Cuadros (2016) dentro del transcurso de aprendizaje se deduce también que los estudiantes son más auditivos, así se emplean varios programas de música e interacción social, como: Kahoot, Facebook, YouTube, correo electrónico, Twitter, Audacity. Además, estas plataformas o apps, son muy factibles de manipular, tanto para los adultos como para los niños, solo se debe comprobar la activación de los mismos y su conexión a internet. Las aplicaciones en los teléfonos sirven para trabajar de forma participativa con los estudiantes.

Por medio de las pizarras digitales se ha alcanzado a captar la atención a los estudiantes, pero es semejante con las redes sociales Facebook, Twitter, Instagram y otras aplicaciones para smartphones WhatsApp, Line, Snapchat, donde se puede aprovechar el impacto de estas. En la antigüedad se estaba acostumbrado a llevar notas en papel, o cuadernos, pero actualmente se puede logar que los estudiantes posean sus textos interactivos y dinámicos, con distintos ejercicios de trabajo para percibir mejor el tema, es significativo anotar que actividades cortas en móviles son efectivas (Torres \& Monreal, 2013). La propuesta innovadora de usar el móvil en la clase de Matemática es factible, ya que hoy en día la mayor parte de personas cuentan con un celular que permita trabajar de forma virtual y dinámica con los estudiantes.

\subsection{Aplicaciones didácticas digitales para matemática}

Cuando se menciona la digitalización, se puede notar una experiencia positiva con el uso de una plataforma adaptativa para matemática (PAM) la que se puede descargar en el celular, es una es una aplicación significativa para el aprendizaje como lo indica Vaillant, Rodríguez \& Bentancor (2020). Este tipo de herramientas ofrece a los estudiantes una oportunidad de instruirse con entusiasmo y eficacia, ya que permite que el estudiante asimile lento o rápido según su ritmo. Es importante brindar a los estudiantes la facilidad de aprender a su ritmo.

Se puede identificar distintos tipos de aplicaciones que ayudarían a crear una solución interactiva que complete el aprendizaje solicitado, se menciona por ejemplo trackback que es un concepto que nace para los weblogs, otro es el Ajax conocido como una técnica para el progreso web de aplicaciones interactivas usando (HTML o XHTML), (DOM y JavaScript), (XML y XSLT). Dentro de las plataformas matemáticas adecuados a los trabajos que se puede efectuar tenemos a la Matlab 
(mathworks), se define como un software de escritorio para la enseñanza del área de matemática (Ascheri, Pizarro, Astudillo, \& García, 2014).

Para Solano, Casas y Guevara (2015) se aprecia el estudio de la plataforma Moodle, como una herramienta de instauración de contenidos muy inteligente y capaz de mejorar los diseños de ambiente, así mismo se trabaja con el modelo TPACK propuesto, es decir se consigue crear una aplicación adaptativa.

La gamificación; como metodología activa, es una opción educativa lúdica que permite a los estudiantes interactuar con una plataforma de juegos, se ha aplicado en distintos campos como escuelas primarias, secundarias, cuidado del medio ambiente etc. Contando con ayuda de diferentes plataformas también encontramos b-learning conocido como un aprendizaje combinado el mismo que tiene como propósito vincular las clases virtuales y presenciales, de la misma forma se proyecta para varias ares de trabajo, la ventaja de este b-learning en que los estudiantes logran desplegar su aspecto cognitivo muy significativo en su incremento académico.

\section{Metodología}

Este es un diseño de revisión sistemática de literatura, en el que se identifica la revisión bibliográfica con relación al tema a tratar, se verifica la evidencia y credibilidad de la temática mediante la utilización de distintos métodos, se confirman datos importantes y verídicos desarrollados anteriormente de forma empírica, toda esta investigación se basa en artículos seleccionados identificando el objetivo de la investigación.

Considerando el análisis de investigación definida para el presente trabajo, el sustento metodológico elegido fue la investigación cualitativa, con la recolección de información a partir de la observación de fenómenos que en este caso se determina al Modelo TPACK y su metodología activa en el área de matemática. Ejemplo de esta metodología se ve en el trabajo de Hoyos (2000), quien establece una investigación documental, en donde aplica los métodos inductivos, en la recopilación y agrupación de datos; en lo particular mediante la unidad de estudios y reflexión, y en lo general lo que corresponde a la procesamiento de datos, de la misma forma el método deductivo de fundamentación en la tercera y cuarta fase que corresponde a la definición de términos y elaboración conceptual del trabajo de investigación, procedente de lo general a lo individual (p. 57)

Para el desarrollo de la investigación se toma como referencia el trabajo documental de Hoyos (2000), donde se resalta cinco fases que son: inicial, descriptiva, interpretativa por núcleo temático, de construcción teórico global y de extensión y publicación (Figura 3). 


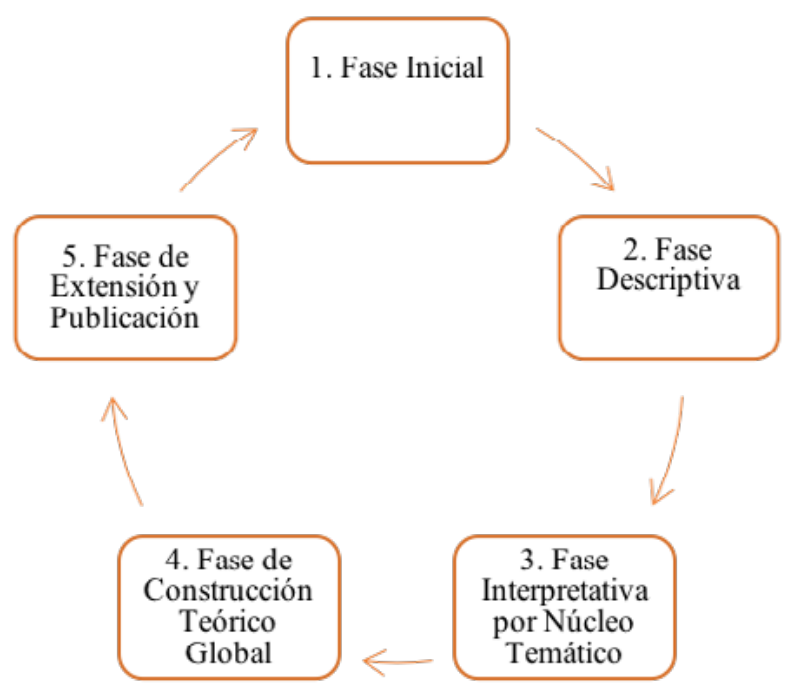

Figura 3. Fases de la investigación cualitativa del TPACK

Fuente: Elaboración propia

A continuación, se detallan cada una de ellas para su proceso investigativo.

- Fase 1 - Inicial: en este punto se ubica el contenido del trabajo de investigación, desagregado en un cuadro de datos definiendo las distintas publicaciones en tecnología y avances del TPACK (Tabla 1), contextualizando los conocimientos del tema que se van adquiriendo, delimitando el espacio del contenido en palabras técnicas, pasos y etapas a seguir, de los cuales se selecciona seis bases de datos académicas. 
Tabla 1.

Bases de datos académicas

\begin{tabular}{|c|c|c|c|c|c|}
\hline No & \multicolumn{2}{|c|}{ Bases de datos académicas } & $\begin{array}{l}\text { Publicaciones científicas y } \\
\text { tecnológicas }\end{array}$ & Documentación & Tiempo \\
\hline 1 & \multirow[t]{2}{*}{$\begin{array}{l}\text { Redalyc: es } \\
\text { un sistema de } \\
\text { información } \\
\text { científica }\end{array}$} & $\begin{array}{l}\text { Artículo Científico de la Revista } \\
\text { Civilizar, Ciencias Sociales y Humanas } \\
\text { https://www.redalyc.org/ } \\
\text { jatsRepo/1002/100264122009/html/index. } \\
\text { html }\end{array}$ & $\begin{array}{l}\text { Competencias TIC de los } \\
\text { docentes de matemáticas } \\
\text { en el ámbito del método } \\
\text { TPACK: evaluación desde } \\
\text { la representación de los } \\
\text { estudiantes. }\end{array}$ & \multirow{4}{*}{$\begin{array}{l}\text { La clasificación } \\
\text { de los temas de } \\
\text { la documentación } \\
\text { de los distintos } \\
\text { artículos, se } \\
\text { realiza por medio } \\
\text { de las palabras } \\
\text { claves TPACK y } \\
\text { TPCK en el área } \\
\text { de enseñanza y } \\
\text { aprendizaje de las } \\
\text { matemáticas y a } \\
\text { fines. }\end{array}$} & 2019 \\
\hline 2 & & $\begin{array}{l}\text { Artículo Científico de la Revista Entre } \\
\text { ciencias: Diálogos en la Sociedad del } \\
\text { Conocimiento } \\
\text { https://www.redalyc.org/ } \\
\text { jatsRepo/4576/457658021003/html/index. } \\
\text { html }\end{array}$ & $\begin{array}{l}\text { Modelo TPACK: ¿Método } \\
\text { para transformar el proceso } \\
\text { educativo considerando la } \\
\text { ciencia } \\
\text { de datos y el aprendizaje } \\
\text { automático? }\end{array}$ & & 2019 \\
\hline 3 & $\begin{array}{l}\text { Scielo: es un } \\
\text { sistema de } \\
\text { información } \\
\text { científica }\end{array}$ & $\begin{array}{l}\text { Artículo Científico de la Revista } \\
\text { Perspectiva Educacional } \\
\text { https://scielo.conicyt.cl/ } \\
\text { scielo.php?script=sci } \\
\text { arttext\&pid=S0718-97292018000200003 }\end{array}$ & $\begin{array}{l}\text { Modelo TPACK como } \\
\text { instrumento de innovación } \\
\text { para el proceso de } \\
\text { enseñanza-aprendizaje en } \\
\text { matemáticas }\end{array}$ & & 2018 \\
\hline 4 & \multirow[t]{2}{*}{$\begin{array}{l}\text { Google } \\
\text { Académico: } \\
\text { es un sistema } \\
\text { que recoge } \\
\text { información } \\
\text { de artículos } \\
\text { científicos, } \\
\text { tesis, } \\
\text { proyectos de } \\
\text { posgrado y } \\
\text { pregrado. }\end{array}$} & $\begin{array}{l}\text { Trabajo de Maestría en Proyectos } \\
\text { Educativos Mediados por TIC. } \\
\text { Universidad de la Sabana; } \\
\text { https://intellectum.unisabana.edu.co/ } \\
\text { handle/10818/33155 }\end{array}$ & $\begin{array}{l}\text { Aplicación del Modelo TPACK } \\
\text { (Comprensión Tecnológica, } \\
\text { Pedagógica y de } \\
\text { Contenido) para Fortificar } \\
\text { la reflexión lógica en los } \\
\text { Métodos de } \\
\text { Enseñanza de las } \\
\text { Matemáticas en el grado } \\
\text { undécimo del Colegio Distrital } \\
\text { Nelson Mandela - Bogotá } \\
\text { D.C. }\end{array}$ & & 2018 \\
\hline 5 & & $\begin{array}{l}\text { Artículo Científico de la Revista } \\
\text { Civilizar: Ciencias Sociales y Humanas } \\
\text { https://revistas.usergioarboleda.edu.co/ } \\
\text { index.php/ccsh/article/view/1106 }\end{array}$ & $\begin{array}{l}\text { Competencias TIC de los } \\
\text { docentes de matemáticas } \\
\text { en el contexto del modelo } \\
\text { TPACK: Evaluación desde } \\
\text { la representación de los } \\
\text { estudiantes. }\end{array}$ & & 2019 \\
\hline 6 & $\begin{array}{l}\text { Scopus: es la } \\
\text { mayor base } \\
\text { de datos de } \\
\text { resúmenes } \\
\text { y citas de } \\
\text { la literatura } \\
\text { examinada por } \\
\text { pares: revistas } \\
\text { científicas, } \\
\text { libros y actas } \\
\text { de congresos }\end{array}$ & $\begin{array}{l}\text { Acta - Comité Latinoamericano de } \\
\text { Matemática } \\
\text { http://funes.uniandes.edu.co/6059/ica } \\
\text { Educativa A. C. }\end{array}$ & $\begin{array}{l}\text { Uso del método TPACK por } \\
\text { estudiantes de un profesorado } \\
\text { de matemática }\end{array}$ & & 2014 \\
\hline
\end{tabular}

Fuente: elaboración propia 
- Fase 2 - Descriptiva: En esta fase se determina el trabajo de campo encontrando en las diferentes dimensiones simplificadas en el cuadro posterior, en donde se representa los distintos tipos de estudio que se han realizado, los referentes y palabras claves del tema de investigación, los principios teóricos, delimitaciones, autores, poblaciones, muestras y métodos que se han estudiado. Esta revisión minuciosa se realizó de acuerdo a lo descrito en la Tabla 2.

Tabla 2.

Fase descriptiva

\begin{tabular}{|l|l|}
\hline Dimensiones & Categorías simplificadas \\
\hline Qué es TPACK & \multirow{2}{*}{ Conceptualización del modelo } \\
\cline { 1 - 1 } Definición & \multirow{2}{*}{ Requerimientos de implementación académica } \\
\cline { 1 - 1 } Características didácticas & \\
\cline { 1 - 2 } Requisitos académicos & Elementos tecnológicos, técnicas \\
\hline Tendencias tecnológicas y pedagógicas &
\end{tabular}

Fuente: Hoyos (2000)

Dentro de la revisión documental se reagrupan las palabras y párrafos claves, seleccionando dichas palabras de la investigación con el resto del contenido, estas palabras fortalecen el estudio y sobresalen en distintos colores para relacionarlos con cada dimensión. Por lo que se determina someter los documentos al análisis cualitativo por el programa N-VIVO11, el cual permite validar las palabras frecuentes y agrupadas que delimitan las dimensiones de análisis.

- Fase 3 - Interpretativa por núcleo temático: en este punto se analiza cada documento para definir y seleccionar los conceptos, se lo divide en un cuadro con las categorías, autores, conceptos, justificación y teorías que amparan los resultados de la investigación del modelo TPACK y su metodología activa en el área de matemática. Del mismo modo, se establece el mismo procedimiento en las lecturas de las seis (6) fuentes de autores representativos de la fase preparatoria que respaldan las dimensiones determinadas en esta investigación (Tabla 3).

Tabla 3.

Interpretativa por núcleo temático

\begin{tabular}{|l|l|l|l|}
\hline Categoría & Autor(es) (año) & Concepto & Justificación \\
\hline Conceptualización & $\begin{array}{l}\text { Ricardo Adán S. } \\
(2019)\end{array}$ & $\begin{array}{l}\text { Su autor argumenta que } \\
\text { el modelo TPACK admite } \\
\text { la edificación de nuevos } \\
\text { instrumentos digitales, } \\
\text { por ende, se recomienda } \\
\text { la utilización del modelo } \\
\text { TPACK en los distintos } \\
\text { programas educativos para } \\
\text { optimizar la enseñanza- } \\
\text { aprendizaje. }\end{array}$ & $\begin{array}{l}\text { Es fundamental que el docente en su } \\
\text { parte profesional obtenga capacidades } \\
\text { tecnológicas, el modelo TPACK permite } \\
\text { unificar pedagogía con tecnología, para } \\
\text { fortalecer los conocimientos que se } \\
\text { debe dominar hoy en día en el aula, } \\
\text { con el propósito de alcanzar resultados } \\
\text { significativos en los estudiantes. }\end{array}$ \\
\hline
\end{tabular}

Fuente: elaboración propia 
- Fase 4 - Construcción teórica global: en esta fase se interpreta cada concepto temático con el fin de identificar estilos, beneficios, vacíos de la investigación, limitaciones, conflictos que presentan la temática estudiada, con la intención de exteriorizar el estado actual de la investigación de una manera global o general, y así poder ubicar nuevas líneas de investigación.

- Fase 5 - Extensión y publicación: en este punto se establece la búsqueda de la mejor estrategia para que el trabajo de investigación sea divulgado, ya sea esta de manera oral o escrita, mediante alternativas como: artículos, seminarios, conferencias, congresos, mesas redondas. El propósito de esta fase es dar a conocer el nuevo conocimiento desarrollado a la colectividad científica y académica.

\section{Resultados}

En la presente investigación, se trabaja el análisis documental, referido sólo al texto, pues la descripción de los conceptos del modelo TPACK, son claros desde cada uno de los autores. Sin embargo, se efectúa el análisis de contenido, con el propósito de cimentar el concepto bibliográfico.

Abarca y Alpízar (2012) en su investigación, plantea el estudio de contenidos desde unos mecanismos y unas fases que admitirán el correcto avance y desarrollo de cada actividad descritas en los siguientes literales:

\subsection{Definir propósito de análisis}

Los documentos, fueron sometidos al análisis cualitativo mediante el programa Nvivo11, teniendo en cuenta la lectura de cada uno y selección de palabras clave que refuerza el estudio de investigación, se identificó de esta manera las palabras frecuentes que definen las dimensiones de análisis.

\subsection{Establecer unidades de observación}

Aquí se despliega la etapa preparatoria, se determinaron los temas de análisis palabras, dimensiones, personajes, ítems, espacios, esta investigación se enfocó en la clasificación de las palabras, pues estas se constituyeron como el centro de apoyo de los criterios y razonamientos de cada dimensión de estudio en relación con su nivel de frecuencia y aparición en cada uno de los documentos y se verificó posteriormente mediante el programa Nvivo11.

\subsection{Clasificación de unidades de análisis}

Dentro de la clasificación y elección de las temáticas de análisis; las palabras diferentes de las demás temáticas de análisis se clasifican mediante el sentido o el término más necesarios que complementen el contexto de la investigación, cotejando con el sentido práctico de la palabra clave tomando en cuenta las pausas y acentos. Del mismo modo, la mezcla de las palabras y su 
representación admite constituir frases u oraciones que forman una expresión fija y, darán como consecuencia una definición propia y concreta (Tomado de: http://definicion.de/palabra/).

\subsection{Construcción de categorías}

En este punto las categorías definidas durante la investigación fueron: definición del modelo; tendencias pedagógicas, exigencias de ejecución académica, tecnológicas. Posteriormente, se validaron las categorías, mediante la identificación por frecuencias de las palabras que definen el modelo TPACK, de acuerdo al rastreo realizado desde software Nvivo11.

\subsection{Procesamiento y análisis de los datos}

Determinadas las dimensiones de análisis, en este punto cada una de ellas fueron ubicadas a un rastreo de frecuencias de palabras, donde se identifica cada uno de los artículos tomados, con la señalización de los aportes y palabras descritas, en ellos, se estableció dar una definición de los propósitos de cada una de las propuestas que se sometieron al estudio de tipo cualitativo acorde a la investigación.

Ordenadas las palabras que se identificaron en la lectura inicial de los artículos, son llevadas al programa Nvivo11, donde se establecen las frecuencias de las palabras, desde su generalización y la relevancia que estas tienen en el presente estudio. Definido el universo de trabajo (documentos), la búsqueda inició con la revisión de los artículos (unidades de análisis) a través del análisis de contenido por medio de resúmenes analíticos especializados - RAE.

Luego, la selección en cada uno de los artículos correspondió a la búsqueda de palabras claves empleadas durante el desarrollo de la investigación. Dichas palabras se enmarcaron en cada uno de los artículos encontrados y permitieron el desarrollo del análisis. Es por eso, que, las palabras empleadas durante la búsqueda de información fueron: TPACK, TPCK, Metodología Activa, conocimiento tecnológico pedagógico del contenido, modelo TPACK o TPCK, TIC, educación virtual, competencias TIC, limitando el universo de búsqueda.

\section{Conclusiones}

EI TPACK es un modelo de enseñanza-aprendizaje para integrar las TIC en la educación de una manera dinámica y precisa. Por ende, es allí donde el docente tiene el reto de dominar los tres conocimientos relacionados, así mismo, el modelo se debe ajustar en el estudiante y en lo que se pretende iniciar. Es indudable que el modelo propone aplicar el constructivismo.

Por otra parte, el docente requiere capacitación permanente por el constante cambio de las tecnologías para adquirir competencias y destrezas en el manejo adecuado de las TIC, también permite que el modelo TPACK sea investigado desde el marco teórico y/o práctico para socializar las experiencias pedagógicas en la comunidad científica y académica. Las demandas de la sociedad requieren una nueva perspectiva de la educación, por eso, es importante estar en formación permanentemente en competencias que fortalezcan metodologías en didácticas activas para el proceso de enseñanza-aprendizaje. El docente se enfrenta a nuevos retos, como: fomentar 
ambientes de aprendizaje que permitan el intercambio y formación de estudiantes reflexivos y críticos mediante la investigación, que demuestren el interés por ilustrarse y construir su conocimiento.

De acuerdo a los resultados establecidos del procesamiento y análisis de dato mediante el programa Nviv011, se pudo determinar y recopilar una selección de artículos y palabras claves que permitieron reforzar el estudio de investigación, de esta manera se incorporó trabajos científicos de renombre académico que permitieron desarrollar un análisis minucioso de información como: modelo TPACK, TPCK, metodología activa, conocimiento tecnológico, pedagogía del contenido, TIC, educación virtual, competencias TIC, detallando y puntualizando así el universo de búsqueda para una mejor comprensión de los procesos educativos y, como ayuda y respaldo para los docentes en su enseñanza de la matemática. 


\section{Referencias}

Abarca, A., \& Alpízar, F. (2012). El análisis de contenido. Capítulo VI. En. Técnicas Cualitativas de Investigación. UCR. Costa Rica, 39-45.

Arévalo, V., García, E., \& Hernández, A. (2019). Competencias TIC de los docentes de matemáticas en el marco del modelo TPACK: valoración desde la perspectiva de los estudiantes. Civilizar: Ciencias Sociales y Humanas, 115.132. https://doi.org/10.22518/usergioa/jour/ccsh/2019.1/a07

Ascheri, A., Pizarro, P., Astudillo, M., \& García, E. (2014). Software educativo en línea para la enseñanza y el aprendizaje de temas de Cálculo Numérico. Revista digital Matemática, Educación e Internet, 14(2). https://doi.org/10.18845/rdmei.v14i2.1662

Cenich, C., Araujo, M., \& Santos, B. (2020). Conocimiento tecnológico pedagógico de contenido en la enseñanza de matemática en el ciclo superior de la escuela secundaria. Revista Perfiles Educativos, X.L.II(167). https://doi.org/10.22201/iisue.24486167e.2019.167.59276

Contreras, Tristancho, \& Fuentes. (2017). Uso de las herramientas informáticas educacionales para la enseñanza de la resistencia de materiales. Revista Virtual Universidad Católica del Norte (50), 299-321. Obtenido de https://revistavirtual.ucn.edu.co/index.php/RevistaUCN/article/viewFile/825/1343

Cueto, M., Morales, C., Burgueño, G., \& Medina, A. (2017). M.A.M.I. DEPORTE: Una metodología activa para la mejora de la iniciación al deporte. Estudio. Journal of Sport and Health Research, 9, 121-128. Obtenido de https://www.researchgate.net/publication/321386415

Deroche, A., Acosta, M., Vegega, C., Tomadon, L., Straccia, L., Pytel, P., \& Pollo Cattaneo, M. F. (2015). Propuesta de desarrollo de aplicación móvil para la evaluación dinámica en asignatura de grado en Ingeniería en Sistemas de Información. In XVII Workshop de Investigadores en Ciencias de la Computación. Obtenido de http://sedici.unlp.edu.ar/handle/10915/46373

Ferreira, E. R., \& Madrigal, T. M. (2014). Desarrollo de aplicaciones móviles para la enseñanza de las ciencias. Revista ReCIBE (1). http://recibe.cucei.udg.mx/index.php/ReCIBE/article/view/26

Gómez, R. (2015). El modelo tpack en los estudios de grado para la formación inicial del prefesarodo en TIC. Didáctica Geográfca (16), 185-201. Obtenido de https://rua.ua.es/dspace/bitstream/10045/65828/1/2015 Gomez-Trigueros Didactica-Geografica.pdf

González, T. C., Montes, d. O., \& Guerrero, L. S. (2018). El análisis didáctico-tecnológico del proceso de enseñanza-aprendizaje de la Matemática. Transformación, 14, 202-213. http://scielo.sld.cu/scielo.php?pi-

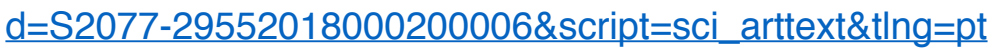


Hoyos, C. (2000). Un modelo para investigación documental. Guía teórico-práctica sobre construcción de Estados del Arte con importantes reflexiones sobre la investigación. Señal Editora., 9-25.

Jiménez, M., Mora, V \& Cuadros, R. (2016). La importancia de las nuevas tecnologías en el proceso educativo. Propuesta didáctica TIC para ELE: mELEndien7dias. Revista Fuentes, 18(2), 209-223. https://revistascientificas.us.es/index.php/fuentes/article/view/2814

Miro, J. (2006). Una metodología activa para la resolución de problemas. Revista XIV Jornadas de ASEPUMA y II Encuentro Internacional.

Muñoz, J., Briceño, E., \& Hernández, J. (2016). Propuesta de clase para la enseñanza de la integral definida con el uso de la tecnología mediante la implementación del modelo Tpack. Revista Electrónica AMIUTEM, IV (1).

Salas, R. R. (2017). Uso del modelo TPACK como herramienta de innovación para el proceso de enseñanza aprendizaje en matemática. Perspectiva Educacional. Formación de Profesores, 52(2), 3-26. https://doi. org/10.4151/07189729-Vol.57-Iss.2-Art.689

Solano, V. C., Casas, D. J., \& Guevara, B. J. (2015). Aplicación Móvil de Realidad Aumentada para la Enseñanza de la Clasificación de losSeres Vivos a Niños de Tercer Grado. Ingeniería, 20(1), 79-93. https://doi. org/10.14483/udistrital.jour.reving.2015.1.a05

Thomas, P. J., Cristina, F., Dapoto, S. H., \& Pesado, P. M. (2017, August). Desarrollo de aplicaciones móviles 3D. In XIX Workshop de Investigadores en Ciencias de la Computación (WICC 2017, ITBA, Buenos Aires). http://sedici.unlp.edu.ar/handle/10915/61957

Torres, O. L., \& Monreal, G. I. (2013). Compartir contenidos educativos con la pizarra digital del aula. Aplicación web "Pizarra digital en casa". Revista Electrónica de Tecnología Educativa (44). https://doi.org/10.21556/ edutec.2013.44.321

Vaillant, D., Rodríguez, Z. E., \& Bentancor, B. G. (2020). Uso de plataformas y herramientas digitales para la enseñanza de Matemática. https://doi.org/10.1590/s0104-40362020002802241 
Copyright (c) 2021 María Fernanda Rodríguez Solís y Santiago Alejandro Acurio Maldonado

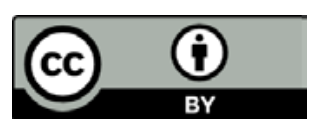

Este texto está protegido bajo una licencia internacional Creative Commons 4.0.

Usted es libre para Compartir-copiar y redistribuir el material en cualquier medio o formato - y Adaptar el documento - remezclar, transformar y crear a partir del material-para cualquier propósito, incluso para fines comerciales, siempre que cumpla las condiciones de Atribución. Usted debe dar crédito a la obra original de manera adecuada, proporcionar un enlace a la licencia, e indicar si se han realizado cambios. Puede hacerlo en cualquier forma razonable, pero no de forma tal que sugiera que tiene el apoyo del licenciante o lo recibe por el uso que hace de la obra.

\section{$\underline{\text { Resumen de licencia - Texto completo de la licencia }}$}

